\title{
Conical diffraction of a Gaussian beam with a two crystal cascade
}

\author{
C. F. Phelan, ${ }^{1}$ K. E. Ballantine, ${ }^{1}$ P. R. Eastham, ${ }^{1}$ J. F. Donegan, ${ }^{1,2, *}$ and J. G. Lunney ${ }^{1}$ \\ ${ }^{1}$ School of Physics, Trinity College Dublin, Dublin 2, Ireland \\ ${ }^{2}$ Principal Investigator, CRANN Research Centre, Trinity College Dublin, Dublin 2, Ireland \\ jdonegan@tcd.ie
}

\begin{abstract}
Internal conical diffraction by biaxial crystals with aligned optic axes, known as cascade conical diffraction is investigated. Formulae giving the intensity distributions for a cascade conically diffracted Gaussian beam are shown to compare well with experiment for the cases of two biaxial crystals with the same and different lengths and with the second crystal rotated with respect to the first. The effects of placing half wave-plates between crystals are also investigated.
\end{abstract}

(C)2012 Optical Society of America

OCIS codes: (050.1940) Diffraction; (260.1440) Birefringence; (260.1180) Crystal optics.

\section{References and links}

1. W. R. Hamilton, “Third supplement to an essay on the theory of systems of rays,” Trans. R. Irish Acad. 17, 1144 (1837).

2. H. Lloyd, "On the phenomena presented by light in its passage along the axes of biaxial crystals," Philos. Mag. 1, 112-120 (1833).

3. L. D. Landau, E. M. Lifshitz, and L. P. Pitaevskii, Electrodynamics of Continuous Media 2nd ed. (Pergamon Press, 1985).

4. M. Born and E. Wolf, Principles of Optics 7th ed. (Cambridge University Press, 1999).

5. J. G. Lunney and D. W. Weaire, "The ins and outs of conical refraction,” Europhys. News 37(3), 26-29 (2006).

6. A. M. Belskii and A. P. Khapaluyk, "Internal conical refraction of bounded light beams in biaxial crystals," Opt. Spectrosc. 44, 436-439 (1978).

7. M. V. Berry, “Conical diffraction asymptotics: Fine structure of Poggendorf rings and axial spike,” J. Opt. A, Pure Appl. Opt. 6(4), 289-300 (2004).

8. M. V. Berry and M. R. Jeffrey, "Conical diffraction: Hamilton’s diabolical point at the heart of crystal optics," Prog. Opt. 50, 13-50 (2007).

9. M. V. Berry, M. R. Jeffrey, and J. G. Lunney, “Conical diffraction: Observation and theory,” Proc. R. Soc. A 462(2070), 1629-1642 (2006).

10. C. F. Phelan, D. P. O’Dwyer, Y. P. Rakovich, J. F. Donegan, and J. G. Lunney, "Conical diffraction and Bessel beam formation with a high optical quality biaxial crystal,” Opt. Express 17(15), 12891-12899 (2009).

11. D. P. O’Dwyer, C. F. Phelan, Y. P. Rakovich, P. R. Eastham, J. G. Lunney, and J. F. Donegan, "The creation and annihilation of optical vortices using cascade conical diffraction,” Opt. Express 19(3), 2580-2588 (2011).

12. V. Peet, "Biaxial crystal as a versatile mode converter," J. Opt. 12(9), 095706 (2010).

13. A. Abdolvand, K. G. Wilcox, T. K. Kalkandjiev, and E. U. Rafailov, "Conical refraction Nd:KGd( $\left(\mathrm{WO}_{4}\right)_{2}$ laser," Opt. Express 18(3), 2753-2759 (2010).

14. M. V. Berry, “Conical diffraction from an N-crystal cascade,” J. Opt. 12(7), 075704 (2010).

15. A. Abdolvand, "Conical diffraction from a multi-crystal cascade: experimental observations," Appl. Phys. B 103(2), 281-283 (2011).

16. M. C. Pujol, M. Rico, C. Zaldo, R. Solé, V. Nikolov, X. Solans, M. Aguilo, and F. Diaz, "Crystalline structure and optical spectroscopy of $\mathrm{Er}^{3+}$-doped $\mathrm{KGd}\left(\mathrm{WO}_{4}\right)_{2}$ single crystals,” Appl. Phys. B 68(2), 187-197 (1999).

\section{Introduction}

Internal conical refraction is a phenomenon of crystal optics in which a beam of light that is refracted into the optic axis of a biaxial crystal propagates as a cone of light in the crystal, and then refracts into a hollow cylinder as it exits the crystal [1-5]. Several attempts have been made to extend the theory of conical refraction to deal with situations involving realistic light beams rather than idealized rays propagating in biaxial crystals. The most fruitful approach is to make the assumption of paraxiality. This approach has led to detailed predictions of the 
intensity distributions of conically diffracted paraxial light beams [6-8]. These predictions have been shown to agree well with theory for the case of the conically diffracted Gaussian beam $[9,10]$. The propagation of paraxial light beams along the optic axes of successive biaxial crystals, known as cascade conical diffraction, has been receiving interest recently for the creation and annihilation of optical vortices [11], as a versatile beam shaping tool [12] and in connection with a novel type of laser based on conical diffraction [13]. Berry has provided a paraxial theory for a general $\mathrm{N}$-crystal cascade in which the relative orientation of crystals of differing lengths is considered [14]. For a two-crystal cascade, conical diffraction leads to a pair of Poggendorf (double) rings, whose relative intensity depends on the angle between the crystals. In the degenerate case, where the crystals are identical the smaller ring becomes a central spot. Abdolvand [15] has provided a first set of experimental results on cascade conical refraction. Here we compare the intensity profiles predicted by the paraxial theory for this case with experiments on crystals of both the same and different lengths. In addition we consider the generalization to include a half-wave plate between the crystals, which can also lead to beams comprising a central spot and an outer ring. Our results demonstrate that the paraxial theory provides an excellent account of the complex beams generated by crystal cascades.

\section{Background theory}

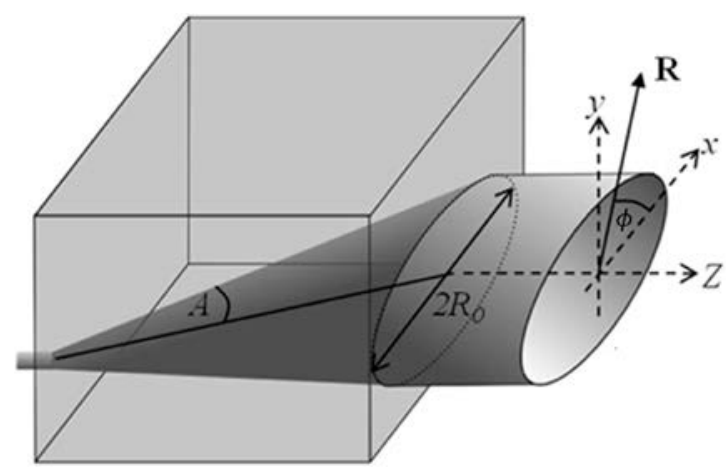

Fig. 1. Schematic diagram showing conical diffraction by one biaxial crystal, and the coordinate system used in the text.

We are considering the case of conical diffraction of a Gaussian beam by propagation along the optic axis of a biaxial crystal of length $l$ and with principal refractive indices $\mathrm{n}_{1}<\mathrm{n}_{2}<\mathrm{n}_{3}$. We define our coordinate system, as shown in Fig. 1, such that conical refraction displaces the center of the incident beam along the $\mathrm{x}$ axis, and measure angles from this axis. A paraxial light beam defined by the Fourier transform of the $\mathrm{x}$ - and $\mathrm{y}$ - components of its electric field $\mathbf{a}(\mathbf{P})$, where $\mathbf{P}=\left\{P, \varphi_{P}\right\}$ is the normalized transverse wave-vector in polar coordinates, is transformed by conical diffraction into:

$$
\mathbf{E}(\mathbf{R}, Z)=\frac{k}{2 \pi} \iint \mathrm{d} \mathbf{P} \mathrm{e}^{i k \mathbf{P} \cdot \mathbf{R}} \exp \left(-1 / 2 i k P^{2} Z\right)\left\{\cos \left(k P R_{0}\right) \mathbf{I}-i \sin \left(k P R_{0}\right) \mathbf{M}\left(\varphi_{P}\right)\right\} \mathbf{a}(\mathbf{P}),(1)
$$

where $\mathbf{M}\left(\varphi_{P}\right)=\left(\begin{array}{cc}\cos \left(\varphi_{P}\right) & \sin \left(\varphi_{P}\right) \\ \sin \left(\varphi_{P}\right) & -\cos \left(\varphi_{P}\right)\end{array}\right)$ and $\mathbf{I}$ is the identity matrix. $\mathbf{R}=\{R, \phi\}$ is the position in the transverse plane and $Z=l+(z-l) n_{2}$ is the propagation distance from the image in the crystal of the incident beam waist (or other reference plane at which $\mathbf{a}(\mathbf{P})$ is specified). $k=n_{2} k_{0}$ is the crystal wave-number with $k_{0}$ being the free space wave number and $R_{0}$ is the radius of the refracted cylinder predicted by geometrical optics. Making the assumption that 
the incident beam is uniformly polarized, i.e., replacing $\mathbf{a}(\mathbf{P})$ with $a(P) \mathbf{e}_{\text {in }}$ where $\mathbf{e}_{\text {in }}$ is the incident polarization vector, and performing the integration over $\varphi_{P}$ we have:

$$
\mathbf{E}(R, \phi, Z)=\left\{\mathrm{B}_{0} \mathbf{I}+\mathrm{B}_{1} \mathbf{M}(\phi)\right\} \mathbf{e}_{\mathrm{in}}
$$

where

$$
\begin{aligned}
& \mathrm{B}_{0}=k \int \mathrm{d} P P \exp \left(-1 / 2 i k P^{2} Z\right) \cos \left(k P R_{0}\right) \mathrm{J}_{0}(k P R) a(P), \\
& \mathrm{B}_{1}=k \int \mathrm{d} P P \exp \left(-1 / 2 i k P^{2} Z\right) \sin \left(k P R_{0}\right) \mathrm{J}_{1}(k P R) a(P) .
\end{aligned}
$$

The generalization to a cascade of several crystals [14] follows on noting that the transformation described by Eq. (1) can be written in Fourier space as

$$
\mathbf{a}(\mathbf{P}) \rightarrow \exp \left(-1 / 2 i k P^{2} Z\right) \mathbf{C}\left(\mathbf{P}, R_{0}\right) \mathbf{a}(\mathbf{P})
$$

where $\mathbf{C}\left(\mathbf{P}, R_{0}\right) \equiv \cos \left(k P R_{0}\right) \mathbf{I}-i \sin \left(k P R_{0}\right) \mathbf{M}\left(\varphi_{p}\right)$.

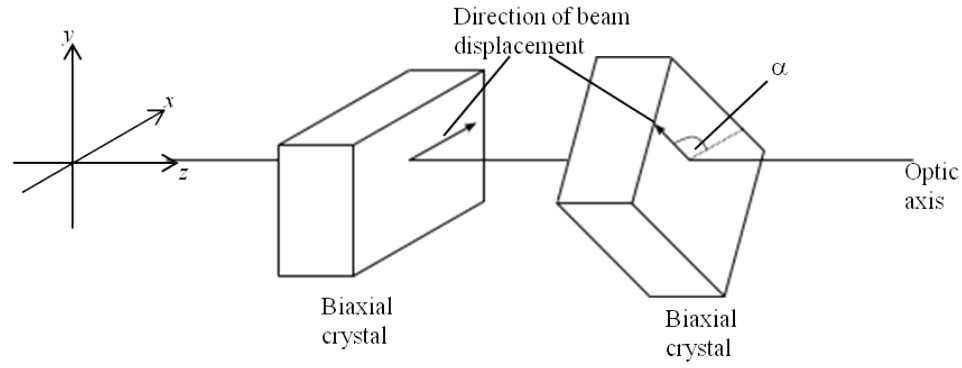

Fig. 2. Cascade conical diffraction setup consisting of two biaxial crystals in series with their optic axes aligned and a relative rotation of one about the optic axis.

We now consider a configuration in which there are two biaxial crystals and the second crystal is rotated about the optic axis by an angle $\alpha$ relative to the first, as shown in Fig. 2. Note that such a rotation displaces the beam, because conical diffraction induces a translation of the center of the beam in the transverse $(x, y)$ plane (Fig. 1), and if the crystals are rotated, so are the displacements induced by the two crystals (Fig. 2). In what follows we will ignore this effect and consider the position of the center of the beam being described to be at the origin of the coordinate system. The crystals are assumed to have equal principal refractive indices and hence equal cone refraction angles. The crystals have lengths $l_{1}$ and $l_{2}$ and geometrical ring radii $R_{1}$ and $R_{2}$. The transformation generated by the second crystal is Eq. (5) with $\quad \mathbf{C}\left(\mathbf{P}, R_{0}\right) \rightarrow \mathbf{R}(\alpha) \mathbf{C}\left(\left\{P, \varphi_{p}-\alpha\right\}, R_{2}\right) \mathbf{R}(-\alpha)$, with rotation matrix $\mathbf{R}(\alpha)=\left(\begin{array}{cc}\cos \alpha & -\sin \alpha \\ \sin \alpha & \cos \alpha\end{array}\right)$. This gives for the final beam

$$
\begin{aligned}
\mathbf{E}(R, \phi, Z)= & \frac{1}{2}\left\{\left(\mathrm{~B}_{0}\left(R_{1}+R_{2}\right)+\mathrm{B}_{0}\left(R_{1}-R_{2}\right)\right) \mathbf{I}\right. \\
& +\left(\mathrm{B}_{0}\left(R_{1}+R_{2}\right)-\mathrm{B}_{0}\left(R_{1}-R_{2}\right)\right) \mathbf{R}(\alpha) \\
& +\left(\mathrm{B}_{1}\left(R_{1}+R_{2}\right)+\mathrm{B}_{1}\left(R_{1}-R_{2}\right)\right) \mathbf{M}(\phi) \\
& \left.+\left(\mathrm{B}_{1}\left(R_{1}+R_{2}\right)-\mathrm{B}_{1}\left(R_{1}-R_{2}\right)\right) \mathbf{M}(\phi+\alpha)\right\} \mathbf{e}_{\text {in }} .
\end{aligned}
$$


The coordinate $\mathrm{Z}$ is now defined by $Z=l_{1}+l_{2}+\left(z-\left(l_{1}+l_{2}\right)\right) n_{2}$ to take into account propagation through the two crystals. If we assume that the two crystals are the same length Eq. (6) reduces to

$$
\begin{aligned}
\mathbf{E}(R, \phi, Z)= & \left\{\mathrm{G}_{0} \times(-\mathbf{R}(\alpha)+\mathbf{I})+\mathrm{B}_{0}\left(2 R_{0}\right) \times(\mathbf{R}(\alpha)+\mathbf{I})\right. \\
& \left.+\mathrm{B}_{1}\left(2 R_{0}\right) \times(\mathbf{M}(\phi)+\mathbf{M}(\phi+\alpha))\right\} \mathbf{e}_{\mathrm{in}}
\end{aligned}
$$

where $\mathrm{G}_{0}$ represents the incident Gaussian beam (taking into account propagation) and we have used the fact that $\mathrm{B}_{0}\left(R_{0}=0\right)=\mathrm{G}_{0}$ and $\mathrm{B}_{1}\left(R_{0}=0\right)=0$.

A half wave plate (HWP) inserted between the crystals has the effect of interchanging the polarisations of the $\mathrm{B}_{0}$ and $\mathrm{B}_{1}$ components before propagation through the second crystal. This interchange of polarisations will occur regardless of the orientation of the HWP. A HWP with its fast axis making an angle $\beta$ with the positive $x$ axis is represented by the Jones matrix $\left(\begin{array}{cc}\cos (2 \beta) & \sin (2 \beta) \\ \sin (2 \beta) & -\cos (2 \beta)\end{array}\right)$. If the HWP is placed between two biaxial crystals with $\alpha$ set to $0^{\circ}$ and with equal lengths $l$ then a beam that propagates through this system is given (in Fourier space) by:

$$
\exp \left(-1 / 2 i k P^{2} Z_{2}\right) \mathbf{C}(\mathbf{P})\left(\begin{array}{cc}
\cos (2 \beta) & \sin (2 \beta) \\
\sin (2 \beta) & -\cos (2 \beta)
\end{array}\right) \mathbf{C}(\mathbf{P}) \mathbf{a}(\mathbf{P}) .
$$

Multiplying out the matrices and integrating over $\varphi_{P}$ leads to:

$$
\begin{aligned}
\mathbf{E}(R, \phi, Z)= & \left\{1 / 2\left(\mathrm{~B}_{0}\left(2 R_{0}\right)+\mathrm{G}_{0}\right) \mathbf{M}(2 \beta)\right. \\
& +\mathrm{B}_{1}\left(2 R_{0}\right) \cos (2 \beta-\phi) \mathbf{I} \\
& \left.-1 / 2\left(\mathrm{~B}_{2}\left(2 R_{0}\right)-\mathrm{G}_{2}\right) \mathbf{M}(2 \phi-2 \beta)\right\} \mathbf{e}_{\text {in }},
\end{aligned}
$$

where

$$
\begin{aligned}
\mathrm{B}_{2}\left(2 R_{0}\right) & =k \int_{0}^{\infty} P \cos \left(2 k P R_{0}\right) a(P) \exp \left(-1 / 2 i k P^{2} Z_{2}\right) \mathrm{J}_{2}(k P R) \mathrm{d} P, \\
\mathrm{G}_{2} & =k \int_{0}^{\infty} P a(P) \exp \left(-1 / 2 i k P^{2} Z_{2}\right) \mathrm{J}_{2}(k P R) \mathrm{d} P, \\
\mathrm{G}_{0} & =k \int_{0}^{\infty} P a(P) \exp \left(-1 / 2 i k P^{2} Z_{2}\right) \mathrm{J}_{0}(k P R) \mathrm{d} P,
\end{aligned}
$$

and we have used the following identities for the integrals over $\varphi_{P}$;

$$
\begin{aligned}
& \int_{0}^{2 \pi} \mathrm{d} \varphi_{p} \exp \left(i k P R \cos \left(\varphi_{p}-\phi\right)\right) \cos \left(n \varphi_{p}\right)=2 \pi\left(i^{n}\right) \mathrm{J}_{n}(k P R) \cos (n \phi), \\
& \int_{0}^{2 \pi} \mathrm{d} \varphi_{p} \exp \left(i k P R \cos \left(\varphi_{p}-\phi\right)\right) \sin \left(n \varphi_{p}\right)=2 \pi\left(i^{n}\right) \mathrm{J}_{n}(k P R) \sin (n \phi) .
\end{aligned}
$$

\section{Experiment compared with theory}

Experiments were carried out to observe the intensity distributions predicted by the theory outlined in the previous section for the cases of two biaxial crystals of equal and unequal lengths. In each case, the biaxial crystals used were slabs of $\mathrm{KGd}\left(\mathrm{WO}_{4}\right)_{2}$ with entrance and exit faces cut perpendicular to the optic axis and with principal refractive indices $n_{1}=2.013$, $\mathrm{n}_{2}=2.045, \mathrm{n}_{3}=2.086$ [16]. The crystals were obtained from Croptics. Figure 3 shows the experimental setup used to observe a cascade conically diffracted Gaussian (CCDG) beam for the case of two identical biaxial crystals. The figure includes an optional half-wave plate between the crystals. The FIP was formed inside the first crystal, as indicated in the diagram,

\#167589 - \$15.00 USD Received 27 Apr 2012; revised 20 May 2012; accepted 21 May 2012; published 25 May 2012 (C) 2012 OSA

4 June 2012 / Vol. 20, No. 12 / OPTICS EXPRESS 13204 
and imaged with unit magnification onto the CCD. The laser used was a $10 \mathrm{~mW}$ He-Ne with a Gaussian intensity distribution. The beam entering the crystal was circularly polarized by a linear polarizer (LP) and quarter-wave plate, and had a minimum beam waist of $40 \mu \mathrm{m}$.

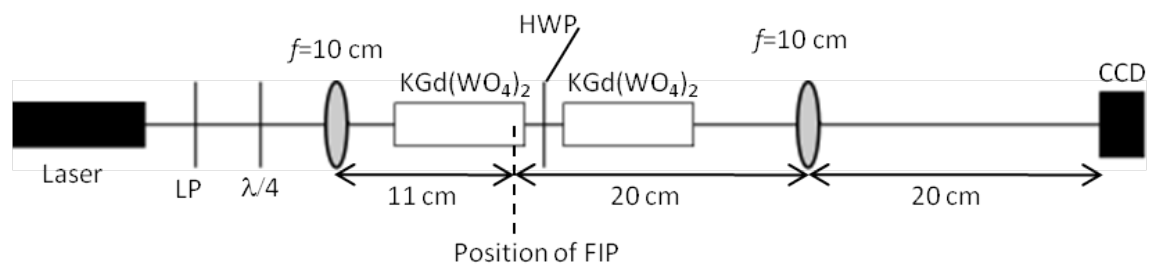

Fig. 3. The experimental arrangement used to observe the intensity profiles in the focal image plane for cascade conical diffraction by a pair of crystals. We consider the effects of rotating one crystal about the optic axis as well as introducing a half-wave plate (HWP) between the crystals.

Figure 4 shows CCD images of the FIP profiles recorded with the experimental setup shown in Fig. 3 without the half-wave plate between the two crystals for four different values of the angle between the crystals, $\alpha$. The predicted increase of intensity of the central spot relative to the ring is evident. Theory is compared with experiment in Fig. 5 for rotations of $15^{\circ}$ and $45^{\circ}$. It is noteworthy that the agreement with theory is less accurate for the smaller rotation angle. This could be because the peak intensity of the central spot grows rapidly relative to the peak intensity of the outer ring as the crystals are rotated away from $0^{\circ}$.

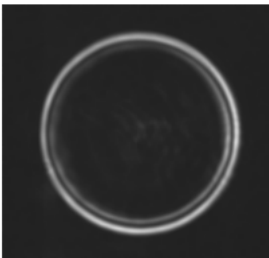

(a)

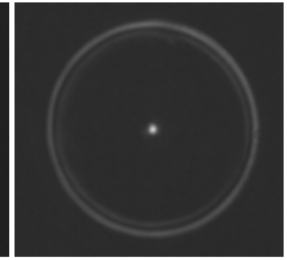

(b)

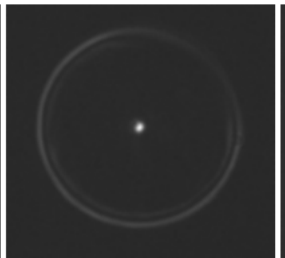

(c)

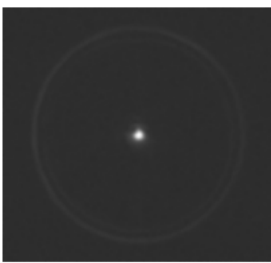

(d)

Fig. 4. Experimental images in the focal image plane for conical diffraction by a pair of crystals with a relative rotation about the optic axis, as shown in Fig. 2. The second crystal is rotated relative to the first by (a) $0^{\circ}$, (b) $15^{\circ}$, (c) $45^{\circ}$ and (d) $90^{\circ}$.

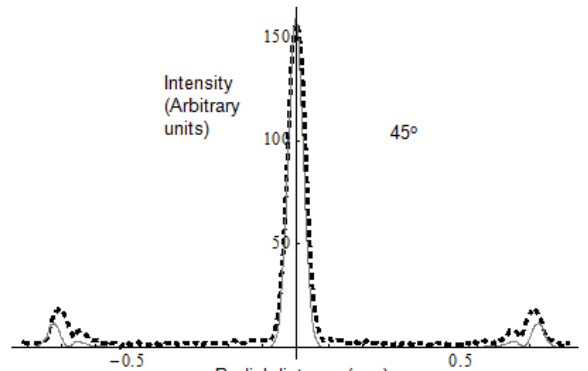

(a)

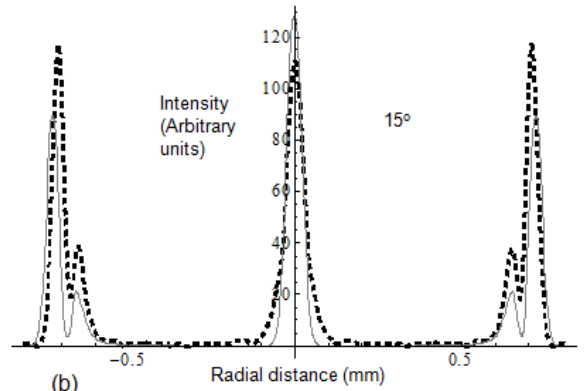

(b)

Fig. 5. Intensity profiles from experiment (dashed line) compared with theory (solid line) for cascade conical diffraction with a relative rotation of $\alpha=45^{\circ}$ (a) and $15^{\circ}$ (b) for the two crystals.

The predictions regarding the effect of placing a half wave plate between the crystals were also confirmed by the experimental setup shown in Fig. 3. The results are shown in Fig. 6. The notable features are a central spot that is elongated in one direction and a ring profile with an angular modulation. The two maxima in the ring's intensity distribution are at the same (diametrically opposite) angular positions as the maxima in the modulated central spot. 


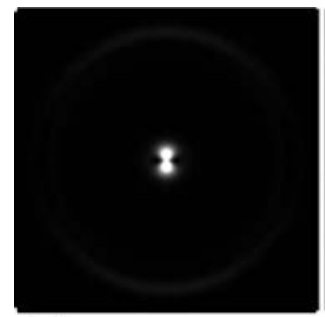

(a)

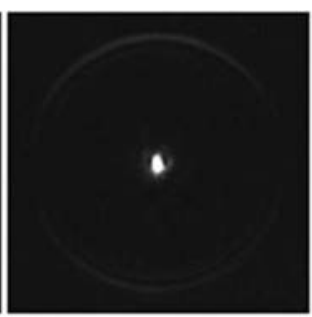

(b)

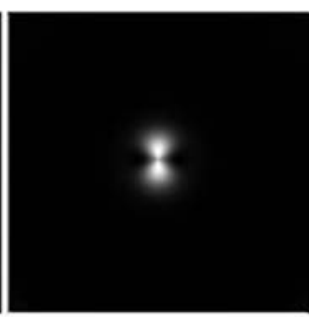

(c)

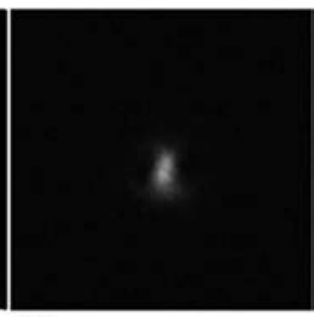

(d)

Fig. 6. Experimental (b,d) and theoretical $(a, c)$ images in the focal image plane following conical diffraction by two identical crystals separated by a half-wave plate (angles $\alpha=\beta=0$ ). Experimental images are obtained from the setup in Fig. 3, and theoretical images calculated using Eq. (9). (c) and (d) are close-ups of the central region of (a) and (b).

A similar experimental setup, shown in Fig. 7, was used to observe the beam produced by propagation through two biaxial crystals with different geometric ring radii. This time the crystal lengths were $3 \mathrm{~cm}$ and $2 \mathrm{~cm}$ respectively. These crystal lengths implied the geometrical ring radii associated with each crystal would be $0.53 \mathrm{~mm}$ and $0.37 \mathrm{~mm}$ respectively. Hence theory predicts that the FIP intensity distribution will consist of two concentric double ring patterns with dark ring radii of 0.9 and $0.16 \mathrm{~mm}$. The same laser source was used in this experiment as in the case of equal length crystals but in this experiment a longer focal length lens was used to form the FIP on the CCD without an imaging lens (this point is discussed in [10]). The minimum waist of the Gaussian formed by the lens was $70 \mu \mathrm{m}$.

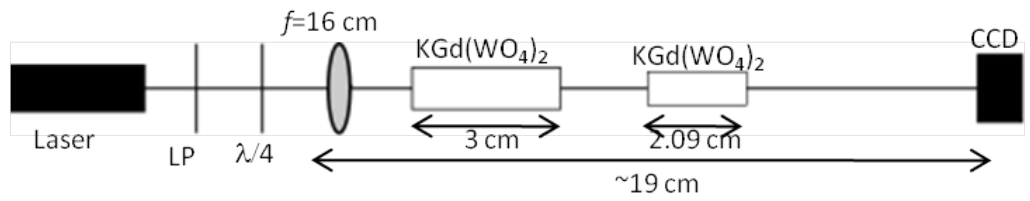

Fig. 7. The experimental arrangement used to observe conical diffraction by a pair of crystals with different geometrical ring radii $R_{0}$.

Figure 8(a) shows a CCD image of the beam recorded using the optical arrangement shown in Fig. 7 with the predicted pair of concentric double rings clearly visible. Theory is plotted against experiment in Fig. 8(b).

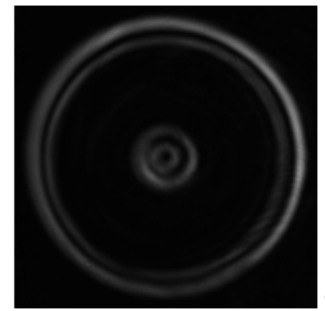

(a)

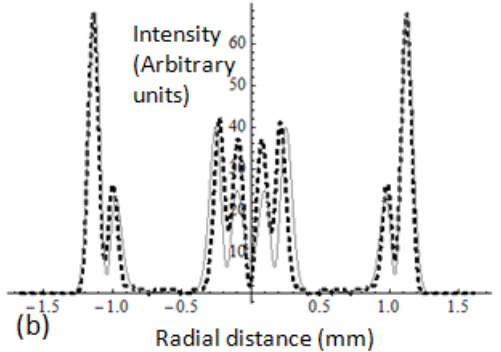

(b)

Radial distance $(\mathrm{mm})$

Fig. 8. (a) Intensity in the focal image plane generated by cascade conical diffraction from two aligned crystals of different lengths, obtained using the apparatus of Fig. 7. (b) Intensity profile from the experiment (dashed line) compared with paraxial theory (solid lines). 


\section{Conclusion}

Paraxial conical diffraction theory can be extended to cases involving more than one biaxial crystal as well as biaxial crystals separated by wave plates in a relatively straightforward manner. The dependence of beam shape on both relative crystal orientation and both polarisation of the incident beam as well as manipulation of the polarisation between crystals facilitates the generation of a wide variety of beam geometries. The dependence of the conically diffracted beams on Gaussian waist and geometric ring radius explored in other publications can be combined with the effects explored in this paper further adding to the versatility of the biaxial crystal as a beam shaping device.

\section{Acknowledgments}

We acknowledge the support of Science Foundation Ireland under research grant numbers 08/IN.1/I1862 and SFI/SIRG/I1592. 